\title{
Frank Hartmann
}

\section{ZUR PHILOSOPHIE DER "WELTCOMMUNICATION"}

Wenn es in der Philosophie einen blinden Fleck gibt - und man hat schon das Körperliche, das Sinnliche, die Gefühle oder das Begehren dafür gehalten dann sind das vor allem die Medien, im Sinne der Materialitäten von Kommunikation, der Technologien von sozialer Synchronisierung und kultureller Tradierung. In Form einer erst im Ansatz fassbaren Kulturmorphologie forderte Marshall McLuhan einst die Auseinandersetzung mit dem technischen Medienwandel und dessen Effekten. Dass eine aus dem Reich der Ideen ausgeblendete Technik stets Begleiter des Gedankens war, wird überdeutlich an den telematischen Medien des 19. Jahrhunderts, mit denen Kommunikation eine wahrhaft monströse Dimension angenommen hat: Weltkommunikation.

Zwar stellt Peter Sloterdijk seine plurale Sphärologie in expliziten Gegensatz zu McLuhan, dessen These vom Global Village er letztlich »ontologische Simplifikation « vorhält. ${ }^{1}$ Dennoch darf McLuhans Ansatz zugute gehalten werden, dass er seiner Zeit - geprägt vom Sputnik-Schock im Jahr 1957, ausgelöst vom ersten globalen technischen Kommunikationssignal des sowjetischen Satelliten - auf Augenhöhe begegnete, während deutsche Meisterdenker noch in spekulativer Befangenheit von einem Seinsgeschick der Technik raunten. Doch bringt das Projekt einer Sphärologie nicht eben auf ähnliche Art zeitdiagnostisches Potenzial mit jenem exzentrischen Impuls zusammen, der schon kennzeichnend war für eine Medienphilosophie, die Philosophieprofessoren konsequent ignoriert haben? Die lassen sich ihre Theorie-Diskurse von Technik und Medien nicht gern verschmutzen. Manchmal jedoch trifft man akademische Außenseiter, die weniger Berührungsängste haben. So stellt sich bei näherer Erkundung heraus, dass es durchaus schon eine zeitgenössische philosophische Reaktion auf die globale Teletechnik und die um 1850 anbrechende Medienmoderne gab - wie Ernst Kapps Philosophie der Technik, auf die die folgenden Überlegungen Bezug nehmen. McLuhan monierte die zweieinhalb Jahrtausende währende Ausklammerung der Technologie aus den Fragen der westlichen Philosophie. Hier aber trat materialiter ein nachmetaphysisches Denken auf, das Mensch und Technik (Natur und Kultur) nicht länger auseinanderdividiert, sondern im Technoimaginären zusammengebracht hat.

\footnotetext{
1 Peter Sloterdijk: Sphären III. Schäume.
}

Frankfurt a.M. 2004, 23 f. 


\section{Projekt Weltmarkt}

$\mathrm{Zu}$ den herausragenden mentalen Effekten der Globalisierung zählt Peter Sloterdijk die Tatsache, dass diese $»$ das anthropologisch Unwahrscheinlichste, das ständige Rechnen mit dem fernen Anderen, dem unsichtbaren Konkurrenten, dem Behälter-Fremden, zur Norm erhoben hat «. ${ }^{2}$ Dieses Unwahrscheinliche besteht zunächst darin, dass Formen der Telekommunikation entwickelt wurden, die in den Jahrhunderten zuvor nicht ganz undenkbar, aber doch technisch unmöglich waren. Erst die technische Nutzbarmachung des Elektromagnetismus ermöglichte jene Mediensphäre, welche die Bedingungen menschlichen Daseins auf dieser Welt neu definiert hat. Telegraphenkabel wurden verlegt, die Infrastruktur für ein globales Handels- und Kommunikationsnetz wurde geschaffen. Während die transzendentalen Kategorien einer reinen Vernunft unter den Bedingungen von Medienapparatur und Telekommunikation implodierten, erfuhr das telematische Projekt seine diskursive Überhöhung - als Weltpost, Weltzeit und Welt-Telegraphenvertrag wurde nun Weltkommunikation einschließlich Welthilfssprachen und Weltpostkarten hergestellt.

Weltprojekte. Wenn Sloterdijk mit dem Kunstbegriff Weltinnenraum Rilkes literarische Metapher entlehnt, um aufzuzeigen, dass Außen- und Innenwelt menschlichen Daseins in ein neues Verhältnis getreten sind, dann setzt er den Akzent in seiner Publikation nicht zufällig auf ein bauliches Symbol neuer Hegemonie, nämlich den Crystal Palace der Londoner Weltausstellung von 1851. Dieser Kristallpalast im Hyde Park, der die neuesten Errungenschaften der Moderne zur allgemeinen Besichtigung vorstellte, war tatsächlich ein geschichtsträchtiger Ort. Britische Bankiers und Industrielle beabsichtigten, durch Vorführung und Vergleich ihrer Produkte den Wettbewerbsvorsprung des Empire am Weltmarkt abzusichern. Unter den vielen prominenten Besuchern befand sich auch der britische Mathematiker Charles Babbage, Konstrukteur programmierbarer Calculating Machines. Von ihm stammt ein detaillierter Bericht von jener Weltausstellung, der sich auf die industriellen Potentiale konzentriert. ${ }^{3}$

Babbage war äußerst verärgert. Wie dann noch in seinen Lebenserinnerungen nachzulesen ist, giftete er offenbar fürchterlich über die Ignoranz des britischen Ausschusses für die internationale Ausstellung, in die sein Difference Engine nicht aufgenommen wurde - und damit der seiner Meinung nach »größte intellektuelle Triumph ihres eigenen Landes «. ${ }^{4}$ Was Babbage bereits

\footnotetext{
2 Peter Sloterdijk: Im Weltinnenraum des Kapitals. Für eine philosophische Theorie der Globalisierung. Frankfurt a.M. 2005, 221.

3 Charles Babbage: The Exposition 1851. Views of
}

the Industry, the Science, and the Government of England. London 1851 (Nachdruck 1968). 4 Charles Babbage: Passagen aus einem Philosophenleben. Berlin 1997, 103. 
ein Jahrhundert vor Alan Turing wusste, war, dass Maschinen programmiert werden können, um Berechnungen zu automatisieren. Er wusste jedoch nicht, dass all dies einst mit einer anderen Technik zur Produktivkraft der Globalisierung zusammenwachsen würde, für die 1851 ebenfalls eine Zäsur bedeutete - die elektrifizierte Nachrichtentechnik. Am 13. November 1851 begann die Brett's Telegraph Company mit einem elektrischen Telegrafenkabel zwischen Dover und Calais den kommerziellen telegrafischen Überseeverkehr.

Noch im selben Jahrzehnt sollte das erste Transatlantikkabel verlegt werden. Es war diese Tele-Technologie, die einer neuen Geopolitik entsprach: Mit ihrer Hilfe gelang es den Briten, in den Jahrzehnten bis zur Jahrhundertwende die erste globale Telekommunikations-Hegemonie zu errichten - in klarer Überlegenheit vor den Amerikanern und den Deutschen. 5 Zwar leitete ein Amerikaner (Cyrus Field) die Transatlantikverkabelung, aber es war ein britisches Unternehmen, das zwischen England und New England die Fernkommunikation ermöglichte; ebenso legte ein Deutscher (Werner von Siemens) die $18.000 \mathrm{~km}$ Kabel nach Indien, aber im britischen Auftrag der Imperial and International Communications, die ab 1934 dann Cable $\&$ Wireless hieß. Die Karte britisch kontrollierter Kabelverbindungen gegen Ende des 19. Jahrhunderts zeigt London als Zentrum eines telegraphischen Welt-Kabelnetzes. Der technolibertäre Mythos vom amerikanischen Informationszeitalter war so gesehen eine ideologische Spätgeburt, explizit gemacht erst 1994 durch den Vorschlag des damaligen Vizepräsidenten Al Gore, die nationalen Data Highways zur Kommunikations-Infrastruktur der globalen Datenströme in Interconnected Networks auszubauen. ${ }^{6}$ In gewissem Sinn bestand dies alles schon seit mehr als hundert Jahren, als der Weltmarkt online ging.

McLuhan sollte an der elektrischen Telegrafie eine Art organischen Zusammenhang diagnostizieren, eine Interdependenz auf Grundlage instantaner elektrischer Informationsbewegung. Den Telegrafen bezeichnet er als Gesellschaftshormon. ${ }^{7}$ Nun sind Hormone Botenstoffe, die in lebendem Gewebe wirken; man kann sie auch als Elemente in organischen Feedbacksystemen verstehen. Tatsächlich wurden die Telegraphendrähte ja in zeitgenössischen Kommentaren als die Nerven der Menschheit interpretiert. Damit ginge es nicht um die Übermittlung von einzelnen Botschaften, sondern um das Funktionieren eines größeren Ganzen bis hin zur Emergenz eines höheren kollektiven Bewusstseins - ein veritables World Brain, wie es H.G. Wells dann deuten sollte. Teletechnologien, und das macht nicht erst die penetrante Community-

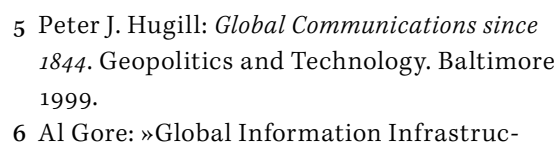

5 Peter J. Hugill: Global Communications since 1844. Geopolitics and Technology. Baltimore 6 Al Gore: »Global Information Infrastruc-

\footnotetext{
ture«. Rede, gehalten anlässlich der ITUKonferenz, Buenos Aires, 21. März 1994. 7 Marshall McLuhan: Understanding Media. The Extensions of Man. New York 1964, 246.
} 


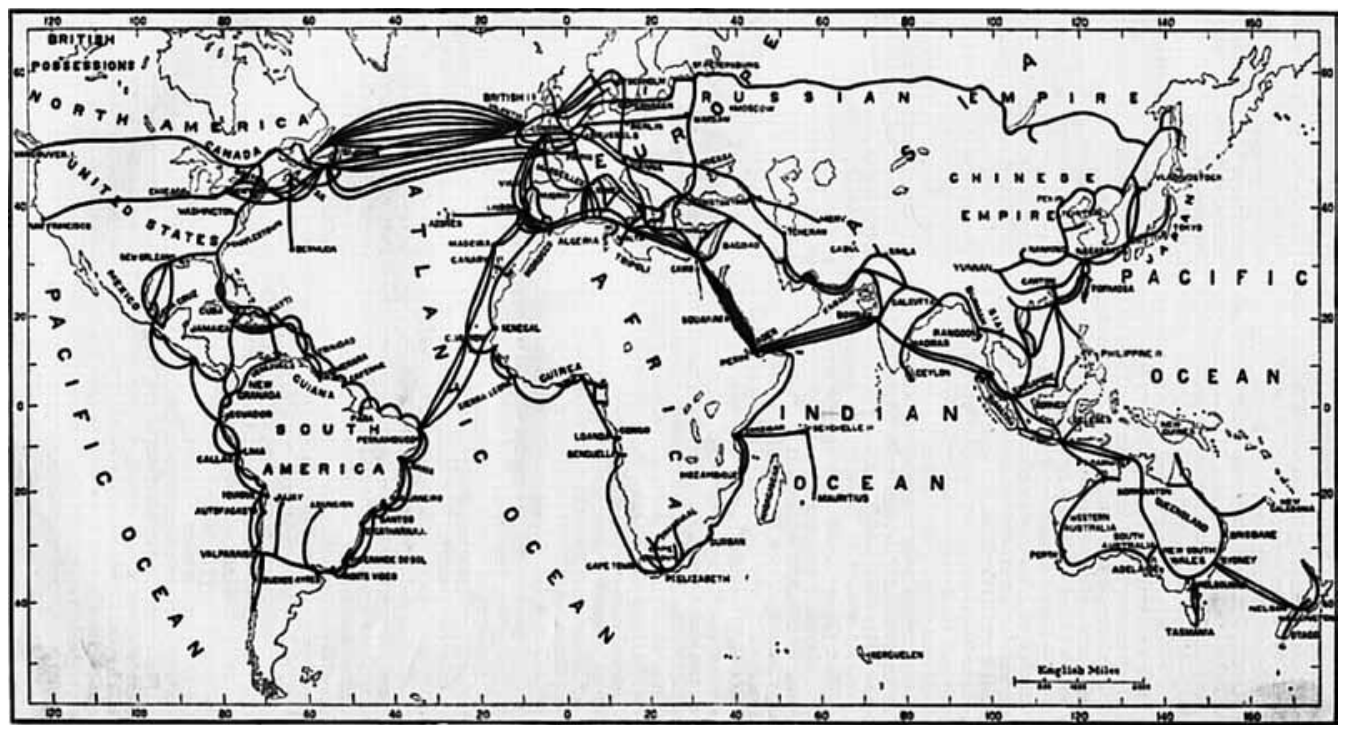

Britisch kontrollierte Seekabel-Verbindungen gegen Ende des 19. Jahrhunderts

Rhetorik des Internets deutlich, unterstreichen in welcher Form auch immer die Tatsache der menschlichen Ko-Existenz. Zu ihren herausragenden mentalen Effekten zählt eben, dass sie das anthropologisch Unwahrscheinliche wahrscheinlich machen: Fernanwesenheit.

\section{VERKEHRS-MENSCHHEIT}

Wie das publizistische Interesse vor allem an den transatlantischen Kabelprojekten von 1857 bis 1866 belegt, war das Bewusstsein für den technischen Fortschritt durch die moderne Kommunikations-Infrastruktur der elektrischen Telegraphie ziemlich ausgeprägt. Um Verständigung ging es dabei kaum; Telekommunikation festigte politische Vormachtstellung und versprach wirtschaftliche Prosperität, da sie die Handelsmärkte mit der Internationalisierung von Angebot und Nachfrage neu definierte. Der schnellere Informationsfluss bedeutete eine unmittelbarere Angleichung von Preisen. Der Telegraph lieferte aktuelle Informationen über den Zustand der verschiedenen Märkte und erlaubte entsprechend rasche Reaktionen: Kommunikation rechnete sich durchaus. Die Teletechnologien fügten sich mithin in das Konzept der liberalistischen Wirtschaftstheorien, welche - wie schon bei Adam Smith belegt - die Wichtigkeit guter Transportmöglichkeiten als Entwicklungsbedingungen für den Markt hervorhoben. Die Existenz funktionierender Kommunikationsbeziehungen war essentiell für die Herausbildung von flexiblen Märkten ohne festen Marktort: der internationale kapitalistische Geldmarkt wurde damit erst möglich. Ebenso wurde die Nachricht selbst zur Ware - 
neben den Börsen profitierten die Zeitungen am meisten von der neuen Form von Aktualität.

So definierte die internationale Telegrafie in der zweiten Hälfte des 19. Jahrhunderts jenen neuen Markt, der sich durch die Beschleunigung des Nachrichtenverkehrs und die aufgrund der Anbindung des Handels an die Informationstechnologie gestiegenen Möglichkeiten nunmehr als Weltmarkt formieren konnte. Gerade seine räumliche Unbestimmtheit macht diesen Weltmarkt zu einem wesentlichen Faktor liberalistischer Theorien; dabei geht es weniger um ein Verschwinden des Raumes, wie es im Zusammenhang mit den Teletechnologien oft beschworen wird, sondern um eine Neubestimmung des Raumes als Bezugsraum globaler Datenströme. So werden Weltmarkt und Weltverkehr, die Kommunikations- und Handelswege der zivilisierten Welt im 19. Jahrhundert zur Deckung gebracht. Die Seehäfen des Welthandels, wie San Francisco, New York, London etc., sind auch die wichtigsten Relaisstationen der soeben entstehenden Weltkommunikation. Das Netz der Telekommunikation wird an jenen Punkten geknüpft, die in merkantiler Hinsicht am interessantesten sind. An fast denselben Knotenpunkten hängen auch heute noch die Datenströme der modernen vernetzten Lebensform, an ihnen wurde verkehrs- und nachrichtentechnisch »eine Welt geknüpft und zusammengezogen, die es vorher nicht gegeben hatte ${ }^{8}{ }^{8}$

So entsteht eben jene Synchronwelt, in der - was mittlerweile zur Alltagserfahrung geworden ist - »die Völker und Kulturen unter Vermittlungszwang geraten sind «, was als längst nicht mehr hintergehbarer Datenozean die Globalisierung erst richtig funktionieren lässt und definitiver Ausgangspunkt einer Theorie des gegenwärtigen Zeitalters wäre. ${ }^{9}$ Während Sloterdijk dieses Thema in die philosophische Arena zurückgeholt hat, scheint für die philosophische Disziplin die Mediensphäre allerdings kein Thema zu sein. Sie zieht sich gern in Begriffswelten zurück. Doch als einst unbekannte Theorie-Objekte sind Medien inzwischen Realität auf technischer und keineswegs bloß begrifflicher Grundlage - nicht Metaphern also, sondern audiovisuelle Techniken der Präsentierung und Synchronisierung einerseits, transportierende und transformierende Techniken für Weltverkehr bzw. Weltkommunikation andererseits. ${ }^{10}$ Und so gelangt man unschwer zur Überzeugung, dass es hier neuer Anstrengungen bedarf, um ein Modell für die Kritik global-kultureller Formbildungen zu gewinnen - jener »zweiten Ökumene [einer] realvernetzten, themenbewegten Verkehrs-Menschheit.« ${ }^{11}$

8 Karl Schlögel: Im Raume lesen wir die Zeit. Über Zivilisationsgeschichte und Geopolitik. München 2003.

9 Sloterdijk:Im Weltinnenraum des Kapitals, $217 \mathrm{ff}$.
10 Frank Hartmann: Globale Medienkultur. Technik, Geschichte, Theorien. Wien 2006. 11 Sloterdijk: Im Weltinnenraum des Kapitals, 224. 


\section{ONTOLOGISChE NeRVOSITÄT}

Fraglos definiert die Medienmoderne die Bedingungen der Möglichkeit von Raum- und Zeiterfahrung neu. Kritik im herkömmlichen Sinn, als gegen die Bilder gerichtetes Schriftunternehmen, ist an ihr Ende gelangt. Die neuen Medienphänomene kennen keine transzendental bestimmbare Bedingung der Möglichkeit, denn sie funktionieren aufgrund ausgeklügelter Ingenieursleistungen. Ihre Ästhetik, die in der Oberfläche moderner Interface-Strukturen zu sich selbst kommt, bedeutet eine unvordenkliche Ausdehnung des raumzeitlichen Wahrnehmungsfensters. Medientechniken als Extensions of man, wie McLuhan wusste, sind im emphatischen Sinn die reale Ausdehnung unserer biologischen Existenz. Doch die Kommunikationen des im neunzehnten Jahrhundert neu entstehenden Weltmarktes scheinen von strukturellen Interessen geprägt, an denen unwesentlich blieb, ob sie sich auf persönlicher Ebene wiederfinden ließen. In die Verlegung interkontinentaler Kabel wurde primär nicht investiert, damit Personen miteinander kommunizieren konnten. Diese Möglichkeit war nicht mehr als ein technisch möglicher - und privat nahezu unerschwinglicher - Nebeneffekt. Vielmehr ging es den beteiligten Nationen darum, ihren politischen und wirtschaftlichen Spielraum auszuweiten und abzusichern.

Das Transatlantikkabel schloss hier eine wesentliche Lücke, die zwischen den territorial zum Teil bereits verkabelten Kontinenten Europa und Nordamerika bestand. Die Amerikaner verkabelten Kuba und Mexiko, die Engländer und die Franzosen ihre westindischen Kolonien, Verlegungen von Kabel über die Azoren und die Kapverdischen Inseln erschlossen auch Südamerika und Afrika. Das transatlantische Südnetz wurde 1874 mit einer direkten Kabelverbindung zwischen Europa und Brasilien eröffnet. In den vier Jahrzehnten zwischen dem ersten transatlantischen Kabelprojekt und der Jahrhundertwende wurden mehrere hunderttausend Kilometer Seekabel zu einem weltweiten System verlegt. Obwohl sich damit im Wesentlichen das britische Empire als erste hegemoniale Macht der Telekommunikationen durchsetzen konnte, jubelte das amerikanische Wissenschaftsmagazin Scientific American zum Anlass der transatlantischen Kabelverlegung:

»This is an important triumph of modern engineering enterprise and skill which deserves our admiration. English telegraph engineers deserve great credit for the boldness and enterprise they have exhibited in laying down so many ocean lines. They have made the ocean a highway of thought. ${ }^{12}$ 
Gleichzeitig wurde in den Jahrzehnten bis 1900 der Telegraphenbetrieb auf nationaler Ebene stark ausgebaut und rationalisiert. Das war für die technische Weiterentwicklung der Telekommunikation wesentlich, denn es musste sowohl auf der Verwaltungsebene grenzüberschreitend kooperiert werden - was 1865 im Abschluss eines Welt-Telegraphen-Vertrags gipfelte - als auch auf Ebene des Geräte- und Leitungsbaus ein Modus der Standardisierung gefunden werden, um den reibungslosen Betrieb sicherzustellen. Knapp hundert Jahre nach der Inbetriebnahme der ersten optischen Telegraphenlinie zwischen Paris und Lille durch den französischen Nationalkonvent war es dann möglich geworden, rund um die Welt zu telegraphieren - über Kabel und bald auch über Funkverbindungen.

Die rein technische Übertragung einer Botschaft, die unabhängig vom Boten und körperlos in Minutenfrist um den Globus reist, schafft nicht nur virtuelle Räume - die Terra incognita der Datenströme, den Cyberspace, der, wie der Name schon sagt, ein Steuerungsraum ist, der die ältere historische Raumordnung überlagert. Sie ist auch philosophisch brisant, zeigt sie doch das schleichende Ende der Textokratien an, wie an der Relativierung der statischen Informationsträger abzulesen ist: der Standorte von Bibliotheken und Archiven, mit allen Implikationen, die das für unsere Arbeits-, Lebens- und Kommunikationsverhältnisse hat. ${ }^{13}$ Sloterdijk attestiert der medientechnischen Verdichtung des Erfahrungsraumes jener Zeit eine ontologische Nervosität, die zur Entstehung des Weltsystems auf neuer Grundlage beigetragen habe, einem eigentlich verflüssigten Grund eines maritimen Denkens. ${ }^{14}$ Doch warum den Rahmen ihrer philosophischen Aufarbeitung mit Schopenhauer begrenzen? Warum nicht die Rolle der medialen Apparatur anerkennen, welche die transzendentalen Kategorien der kantischen Philosophie damals aufzulösen begann? Nur weil Telegraphie und Fotografie - und bis heute allgemein: Medien - in den philosophischen Studierstuben grundsätzlich außen vor zu bleiben hatten? Sehen wir zu, ob sich hier nicht noch ein anderes Bild gewinnen lässt.

\section{Artefaktische Aussenwelt}

Materialitäten der Kommunikation und generell die perzeptuelle Synthesis neuer technischer Apparate setzten das philosophische Konzept der transzendentalen Subjektivität in Parenthese. ${ }^{15}$ Die globale Medientechnik steht für das Veralten jener philosophischen Denkfigur, die als subjektzentrierte Bewusstseinsphilosophie gilt. Hier kommt der vergessene Philosoph Ernst Kapp ins Spiel - keine große Figur der jüngeren Philosophiegeschichte, aber wir verdan-

13 Michel Serres: Atlas. Paris 1994.

14 Sloterdijk: Im Weltinnenraum des Kapitals, Kap.16.
15 Jonathan Crary: Aufmerksamkeit. Wahrnehmung und moderne Kultur. Frankfurt a.M. 2002, 23. 
ken ihm die erste explizite Philosophie der Technik, die ihre Spuren im Denken und in der Terminologie bei Freud, Heidegger und auch noch Teilhard de Chardin und Marshall McLuhan hinterlassen sollte. Ich wage sogar die These, dass Kapp am Beginn einer neuen Zeit des Philosophierens steht, die deshalb neu ist, weil sie beginnt, ein Technoimaginäres in ihr Denken aufzunehmen, aber eben nicht als Ausbuchstabieren irgendeines Seinsgeschicks der Technik, sondern als Auseinandersetzung mit der Wirklichkeit apparativer Synthesis.

In seiner Sphärologie zeichnet Sloterdijk jenen Weg nach, der von mikrosphärischer Intimität über makrosphärische All-Einheit zu einer polysphärischen Verfassung des Weltganzen führt. Diese Philosophie stellt den Menschen als den Effekt eines selbst erzeugten Raumes dar, der ihn zunehmend in ein Verhältnis mit dem Weltganzen bringt. $\mathrm{Zu}$ diesem Sphärischen der Existenz, das auf mehreren Ebenen (Blasen, Globen, Schäume) lokalisierbar ist, gehört eben jene Mediensphäre, die seit Mitte des 19. Jahrhunderts auf Basis elektromagnetischer Anwendungen ausgebaut wird. Das Mediale beinhaltet einen kommunikativen Imperativ, der über die Vielheit der Eigensinnigkeiten das homogenisierende Ideal des eingangs erwähnten ständigen Rechnens mit dem fernen Anderen legt - ein Rechnen im buchstäblichen wie im übertragenen Sinne.

Die techné solchen Rechnens - altgriechisch für die Fähigkeit des Hervorbringens, im weiteren Sinn auch für Handwerk - stellt sich neu, weil sich die neuen Apparate und Instrumente von den herkömmlichen Werkzeugen und ihrer Mechanik grundlegend unterscheiden. Das nun ist die These von Ernst Kapps exzentrischer Philosophie der Technik, die den Versuch darstellt, auf Augenhöhe mit den Ingenieuren jener Zeit einen kulturmorphologischen Neuansatz zu wagen. ${ }^{16}$ Kapp suchte dazu die Werkzeug- und Maschinenwelt als Teil der Entstehungsgeschichte menschlicher ${ }^{2}$ Cultur« zu ergründen, und zwar genau zu dem Zeitpunkt, da diese das makrosphärische Ereignis einer $»$ Weltcommunication« hervorgebracht hat. Wenn Kapp die neue »artefactische $\mathrm{Au}-$ ßenwelt« der Technik als eine Projektion und damit als eine Ausweitung der menschlichen Organanlagen thematisiert, dann wird damit im Wesentlichen dieser eine Gedanke ausgeführt, nach dem die mit den Mitteln der künstlichen

16 Ernst Kapp (1808-1896), Anhänger der anti-idealistischen naturwissenschaftlichen Forschung in der Folge Alexander von Humboldts und Carl Ritters, dem Begründer der wissenschaftlichen Geographie, lehrte an einem deutschen Gymnasium Geographie und publizierte zum Thema philosophische Erdkunde. Ab 1849 lebte er mit seiner Familie in einer deutschen Exil-Kommune in Texas, wo er als Farmer und Zimmermann, ja sogar als Wellness-Anbieter (»Badenthal Dr. Ernest Kapp's WaterCure « in Sisterdale, Texas) tätig war. Kapp kehrte später nach Deutschland zurück und publizierte Grundlinien einer Philosophie der Technik. Zur Entstehungsgeschichte der Cultur aus neuen Gesichtspunkten. Braunschweig 1877 (alle folgenden Zitate sind dieser Erstauflage entnommen). 
Elektrizität vorgenommene Restrukturierung der Kommunikationsverhältnisse auf globaler Ebene eine kollektiv implementierte Metaphysik darstellt (Noosphäre, Global Brain etc.).

Das kategorial Andere dieser Technik tritt erst mit der künstlichen Elektrizität zutage. Werkzeuge, so Kapp, sind als Entlastung der organischen Physis entstanden, wobei zwischen Organ und Werkzeug ein koevolutionäres Verhältnis herrscht, das zu einem $»$ Verwachsensein des Werkzeugs mit dem menschlichen Selbst« führt. ${ }^{17}$ Indem die Hand sich mit einem Gegenstand befasst, herrscht eine Rückbezüglichkeit zwischen diesem und der »Mache« der Menschenhand, die Grundlage für jeden Mechanismus wird. Letzterer ist als mechanische Unterstützung von »Hände Werk« deren mittelbare Erweiterung. Diese Erweiterung versteht Kapp nicht als simple Verlängerung, etwa wie wenn ein Stock die Reichweite des Armes verlängert, sondern als »Mittel der Erhöhung der Sinnesthätigkeit«. So findet er zur Bezeichnung der »Organprojection«, wobei dieses Projizieren als ein unbewusstes Entwerfen oder ein unbemerktes Hervorbringen zu verstehen ist. Mehr noch: technische Innovation ist immer von der Verrichtung her zu denken, nicht von der Erfindung her; Tätigkeit und nicht Reflexion ist der Motor des Fortschritts, argumentiert Kapp. Neue Werkzeuge entstehen also nicht durch Nachdenken über Verbesserungen, sondern durch langwierige Optimierung in winzigen Schritten. Diese eindrucksvolle These sollte erst in einer von Rassismen, Biologismen und von dem Geniekult der Erfinder abgelösten Evolutionsforschung bestätigt werden. ${ }^{18}$

Damit stellt sich die Frage nach der Dialektik von Kulturen, die einerseits Traditionen anbieten sollen, um nicht mit jeder Generation am Nullpunkt beginnen zu müssen, und die andererseits auch Spielräume für Neues zuzulassen haben. Als deutscher Emigrant in Texas begeisterte sich Kapp für die amerikanische Alltagspragmatik, die solchen Spielraum anscheinend geboten hat. Kapp bezieht sich hierbei auf die Philosophy of the Axe, die nirgends geschrieben steht, sondern die ihm »ein alter Backwoodsman im westlichen Texas (...) vordemonstrirte ${ }^{19}$ Mit ihrem elegant geschwungenen handle sei die amerikanische Axt der einförmigen, kerzengeraden, steifen und störrigen deutschen Axt so überlegen, dass man mit ihr das Doppelte und Dreifache einer Tagesarbeit schaffe. Die neue Form sei entstanden als Anpassung an die in Texas vorkommenden Holzarten, für welche sich die deutsche Axt offenbar als deutlich weniger geeignet erwiesen hat.

17 Kapp: Grundlinien einer Philosophie der Technik, 4o f. - Hier der Verweis darauf, dass Organon im Altgriechischen sowohl für »Körperglied« wie auch für dessen Nachbild, das »Werkzeug«steht.
18 André Leroi-Gourhan: Hand und Wort. Die Evolution von Technik, Sprache und Kunst, Frankfurt a.M. 1995 ('1964).

19 Kapp: Grundlinien einer Philosophie der Technik, 241. 


\section{BEFREIUNGSGESTEN}

Weder gibt es eine von Technik reine Kultur noch eine von kulturellen Eigenheiten freie Technik. Technik und Kultur bilden keine unmittelbaren Gegensätze, sie bedingen und überformen sich wechselseitig. Vom Spielraum ihrer wechselseitigen Aneignung hängt ab, ob eine Technik die Funktionszusammenhänge in einer Kultur erweitert oder abschließt, ob sie bloß stehende Formen tradiert oder neue Formen generiert. Kapp hat nicht technokratisch argumentiert, wohl aber im Sinne eines optimistischen Systemprinzips und eines fortschreitenden Kollektivbewusstseins der menschlichen Gattung. Immerhin bleibt aus seiner Philosophie der Technik zu lernen, dass technologische Innovation grundsätzlich als Befreiungsgeste fungiert. Für Kapp erstrahlt an der modernen Technik kein aufgeklärtes Subjekt, sondern immer »nur der Abglanz aus der Tiefe des Unbewußten « ${ }^{20}$ Denn auf der sinnlichen Wahrnehmungsebene bleibe den Menschen jene Dimension der Technik eigentlich unbegreifbar, die nichts anderes wäre als eine Art Rückkopplung eines Teils der Natur mit sich selbst. So würden sich schon seit Urzeiten Werkzeuge und Menschen eher finden, als dass diese jene erfinden würden. Die Strukturähnlichkeit von Organischem und Technischem, auch nicht gerade ungewöhnlich für jene Zeit, führte Kapp zu einem Vergleich der Querschnittsfläche eines Tiefseekabels mit der eines menschlichen Nervs: $\gg$ Die Nerven sind Kabeleinrichtungen des thierischen Körpers, die Telegraphenkabel sind Nerven der Menschheit! $\ll^{21}$

Mit dieser Analogisierung von Nerven und Telegraphen bezog sich Kapp u. a. auf die Cellularpathologie des Berliner Mediziners Rudolf Virchow und auf die ganzheitliche Physiologie des Naturphilosophen Carl Gustav Carus. Die im 19. Jahrhundert verbreitete Parallelisierung zeigt an, dass die Zeit der aufstrebenden Naturwissenschaft und der sich ausbreitenden Medienkultur nach einem Weg suchte, die Konstruktionen einer zweiten Natur im Wechselverhältnis mit den Bedingungen der ersten Natur zu begreifen. Die Theorie der organischen Entwicklung, unter dem Eindruck von Charles Darwins Lehre, und die mechanisch-industrielle Praxis suchen nach ihrem Grund. Diese Suche hat mehr als es auf den ersten Blick scheinen würde mit den Wissenschaften vom Menschen zu tun; doch erst etwa ein Jahrhundert später, bei Michel Foucault, schien es möglich, neben das formal-philosophische ein historisch-technisches Apriori zu setzen. Mensch und Technik sind epistemisch nicht klar abzugrenzende Gebiete eines Diskurses, der zu Kapps Zeit seinen Ort definitiv noch nicht gefunden hatte. Und doch stand seine Argumentation klar und

2o Kapp: Grundlinien einer Philosophie der Technik, 113, 123.
21 Kapp: Grundlinien einer Philosophie der Technik, 141. 

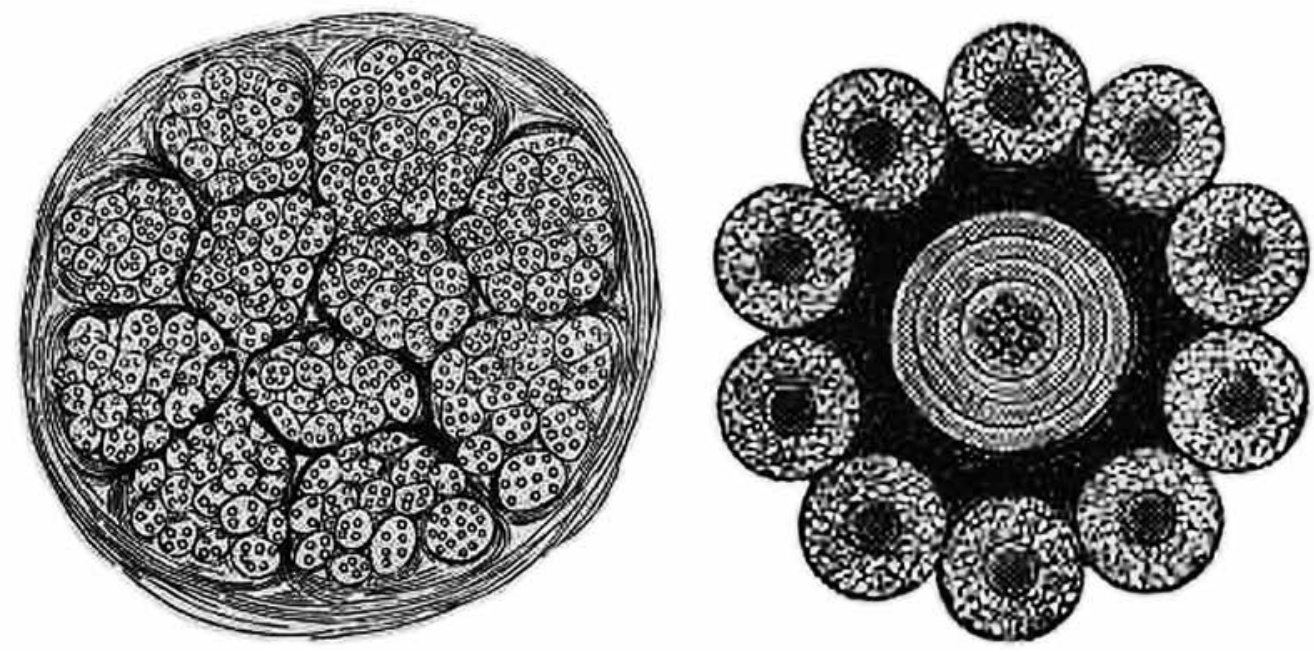

Querschnitt eines Seekabels und eines menschlichen Nervenstrangs

deutlich auf Seiten technischer Hervorbringungen, die - als unbewusstes Nachbild - die Geheimnisse der organischen Innervation erschließen. So wie ihm der Mensch nicht als losgelöster Geist, sondern nur als die eingefleischte Seele wirklich war, so mussten ihm die Telegraphie und im Besonderen das Transatlantikkabel als Nervenleitungen erscheinen, deren Körper soeben als Weltgesellschaft in Formung begriffen war.

Kapp argumentierte vor dem Hintergrund der Frage nach verborgenen Entwicklungsgesetzen des Technischen und der Selbstorganisation des Sozialen. Der mögliche Vorwurf, hier werde Technik biologisiert, beruht auf einem Missverständnis. Als erst retrospektiv erkennbare Nachformung, der alle Technikentwicklung entspricht, ist die Organprojektion gleichsam Realisierung eines technisch Unbewussten, das von den Grundformen der Werkzeuge bis hin zur Atlantikverkabelung am Werke ist und von dem »der Mensch sich Vorstellungen nur auf Kosten der Integrität seines Selbstbewußtseins « mache. ${ }^{22}$ Das ist philosophische Bescheidenheit. Diese verführte Kapp jedoch nicht zum Technikdeterminismus, sondern zum Ansatz einer - nicht mehr wirklich ausgeführten - Kulturmorphologie. Sollte Technik einem bislang unentdeckten Muster der biologischen Evolution entsprechen? Den Kulturapokalyptikern wäre damit der Wind aus den Segeln genommen worden; Kapp wurde vergessen, doch seine Philosophie der Technik stand noch lange in den Regalen und ihre Frage blieb bis in die 1950er-Jahre hinein virulent, als großzügig verunglückte Modelle einer »Planetarisierung des Menschen« den Reigen einer spirituell überhöhten Vernetzungsideologie eröffneten. ${ }^{23}$ 


\section{TRANSANTHROPOLOGISCHER RAUM}

Die Philosophie der Gegenwart ist nicht nur in dieser Hinsicht durch eine verpasste Chance gezeichnet. Sie ließ es nicht zu, dass ihr humanistischer Ansatz und ihre hermeneutische Methodik durch die Logik des Technischen verunreinigt werden. Deshalb ist sie auch in weiten Teilen derart schal, uninspiriert und folglich unbedeutend geworden. Andererseits - und abschließend bemerkt - hat Peter Sloterdijk seit den 1980er Jahren eine ganze Generation dafür sensibilisiert, dass im Philosophischen auch anderes möglich ist: problembezogenes Denken und anspruchsvolle Subversion. In seiner Sphärologie schlug er das schöne Konzept vom »Seelenraumteiler« vor. »Alle Geburten sind Zwillingsgeburten «, heißt es in diesem Zusammenhang, und der Weg, auf den uns das bringt, ist die ständige Suche nach dem ergänzenden Anderen. ${ }^{24}$ Eingedenk grundlegender Ergänzungsverhältnisse gilt es hier nicht für Personen, sondern für ganze Diskurse Nachholbedarf anzumelden - etwa an Ergänzungen betreffend Kultur und Technik gerade zum Zeitpunkt ihres Verschwindens hinter interaktiven Oberflächen und hochkomplexer Software, in virtuellen Räumen voll programmierter Multimedia-Objekte.

Es sollte zu denken geben, dass Kommunikationsverhältnisse nicht einfach aus kommunizierenden Subjekten bestehen, die irgendwo an einem Tisch zusammen oder am Ende irgendeiner Leitung sitzen. Nichtmenschliche Agenten der Transformation kommen immer mehr ins Spiel, ebenso unbemerkte Infrastrukturen und semantische Technologien, die nicht wahrnehmbare Ebene der Metadaten. Wir sind längst dabei, die Objektwelt in unsere Datenwelt zu integrieren. Wird es dabei denn nicht zunehmend sinnlos, diese Orte der KoExistenz nach Sein und Schein auseinanderzudividieren? Zeichnet sich hier nicht eine neue Ontologie des transanthropologischen Raumes ab? Wenn dem so ist, warum dann nicht über die kanonischen Texte hinausgehen, neue Lektüren wagen, posttraditionale Medienkunde betreiben, konzeptuellen Modellen nachspüren und Terminologien entwickeln, um die Bedingungen technischer Möglichkeiten explizit zu machen. Ein solches Explizitmachen, gerade auch als aktive Umgestaltung von Diskursen, führen Sloterdijks Schriften exemplarisch vor.

22 Kapp: Grundlinien einer Philosophie der Technik, 161.

23 Pierre Teilhard de Chardin: Le Phènomene humain. Paris 1955.
24 Peter Sloterdijk: Sphären I. Blasen. Frankfurt a.M. 1998, 419. 